tests that are particularly difficult to interpret; the six donors described in this report are examples.

We believe that we owe it to blood donors to investigate their repeatedly positive results extensively despite the added expense and the time required. The reasons for repeatedly false positive results in screening tests are largely unknown, but this should not mean that the donors are left unsure as to whether or not they have the HIV infection with all the difficulties associated with this diagnosis. Donors' tangible expression of concern for others should, if at all possible, not be allowed to become a source of worry for themselves ${ }^{4}$; they deserve reassurance whenever possible.

1 HMSO. Guidelines for the blood transfusion service. 2nd edn. London: HMSO, 1993.

2 Anonymous. Interpretation and use of the western blot assay for serodiagnosis of human immunodeficiency virus type 1 infection. MMWR 1989;38:1-7.

3 Anonymous. Acquired immunodeficiency syndrome (AIDS): Proposed WHO criteria for interpreting results from western blot assays for HIV-1, HIV-2 and HTLV-I/HTLV-II Wkly Epidemiol Rec 1990;65(37):281-3.

4 Perry S, Jacobsberg L, Fishman B. Suicidal ideation and HIV testing. $¥ A M A$ 1990;263:679-82.

\title{
Osteolytic bone lesions in a patient with idiopathic myelofibrosis and bronchial carcinoma
}

\author{
D J Clutterbuck, A E Morrison, C A Ludlam
}

\begin{abstract}
A 59 year old man with longstanding myelofibrosis and previous splenectomy was incidently found to have a large lytic lesion in his left femur which required operative fixation. He had undergone right upper lobectomy for squamous carcinoma of the bronchus five years earlier. Histological analysis of bone reamings showed no evidence of metastatic carcinoma. Osteosclerosis is frequently noted in patients with myelofibrosis but osteolytic lesions are uncommon and may be confused with metastatic malignancy.

( $\mathcal{H}$ Clin Pathol 1995;48:867-868)
\end{abstract}

Keywords: Myelofibrosis, bronchial carcinoma, osteolytic lesions.

\section{Case report}

A 59 year old man with stable myelofibrosis of 33 years duration presented acutely unwell with bilateral pneumonia, congestive cardiac failure and acute renal failure. He required intensive supportive care including mechanical ventilation, diuretics, inotropes, and intravenous broad spectrum antibiotics. Bronchial washings grew enterococci on culture. Pneumococcal antigen was negative. He made a slow recovery. During his recovery, the patient complained of weakness and paraesthesia in his left leg. He was found to have a flaccid paralysis of the left foot with absent ankle jerk and plantar reflex and sensory loss of all modalities below the knee.

An $x$ ray of the left femur showed a lytic lesion occupying the mid and lower third of the shaft of the bone with extensive endosteal scalloping (figure). A bone scan showed increased uptake of the isotope at the diaphysis of the femur consistent with a metastatic malignancy or primary bone tumour. As there was thought to be significant risk of fracture through this lesion, intramedullary nailing of the bone was performed. Reamings taken from the femur were examined histologically and found to contain macrophages, lymphocytes and haemopoietic cells but there was no evidence of malignancy. Following surgery, the patient made a good recovery. Nerve conduction studies showed changes attributed to critical illness polyneuropathy.

His past medical history included splenectomy at the age of 45 for massive splenomegaly after several painful splenic infarcts, and a right upper lobectomy for squamous carcinoma of the bronchus when aged 55 years.

A skeletal survey during this admission showed an abnormality of the bony trabecular pattern in the head of the left humerus, thought to be due to fibrous dysplasia, previously noted in 1960 at which time no abnormality of the left femur was reported. No bone scan was done at the time of lung resection.

Six months later, despite regular post splenectomy prophylaxis with penicillin $\mathrm{V}$, the patient developed a fatal pneumococcal pneumonia and septicaemia. A postmortem examination was not performed.

\section{Discussion}

Idiopathic myelofibrosis is a chronic myeloproliferative disorder characterised by marrow 


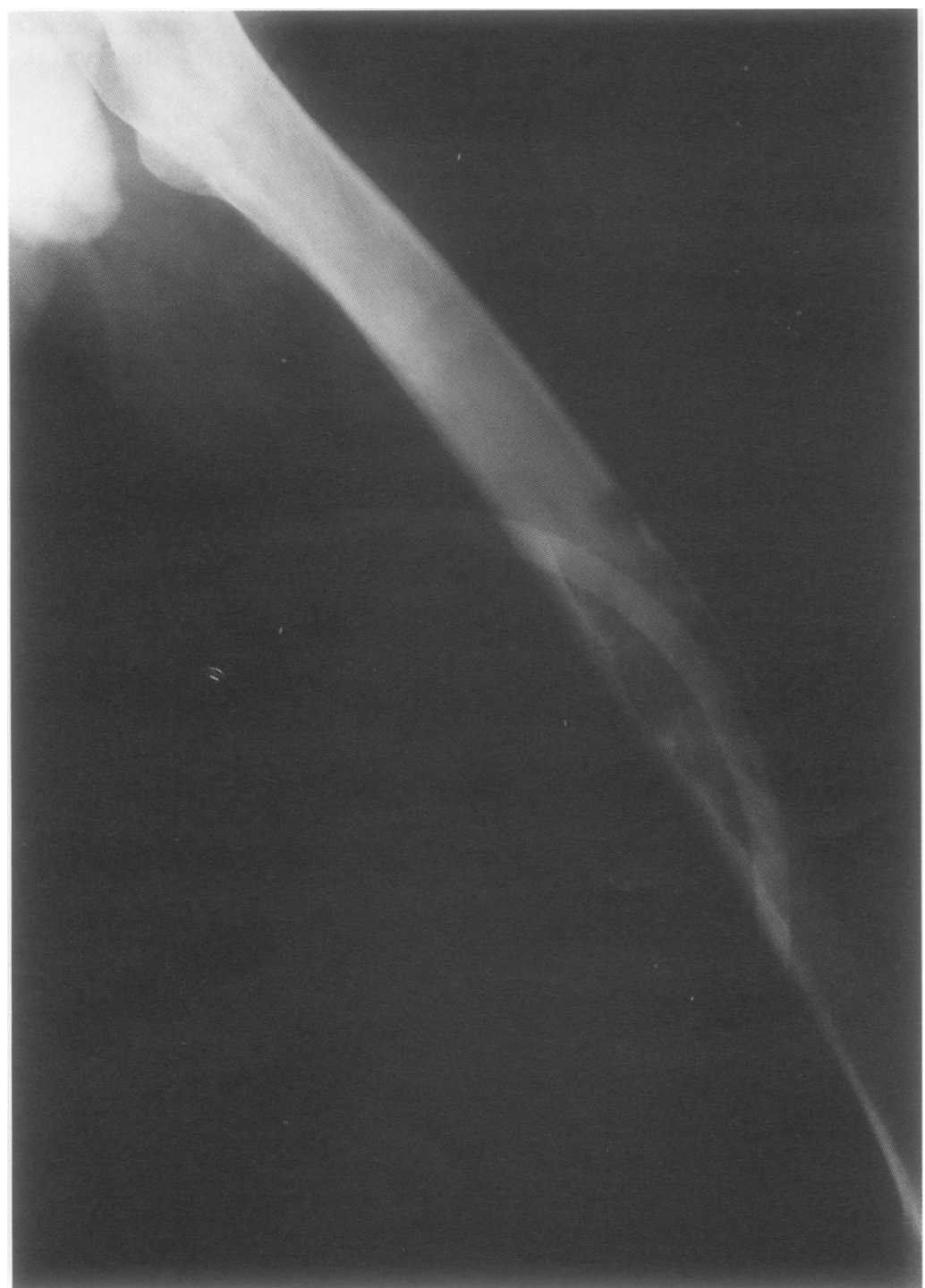

$x$ Ray of femur showing lytic lesion with endosteal scalloping.

fibrosis, splenomegaly and a characteristic appearance of the peripheral blood. Extramedullary haemopoiesis occurs, most commonly in the liver and spleen and less frequently in a variety of other sites.

Bone changes are a characteristic feature of myelofibrosis. Osteosclerosis occurs in 40 to $70 \%^{1-3}$ of patients and the combination of osteosclerosis and splenomegaly is strongly suggestive of myelofibrosis. ${ }^{4}$ Osteolytic lesions, however, are much less common. Osteolytic lesions are frequently diffuse and may be painful. ${ }^{12}$ Reported cases have been pre- dominantly in patients transforming from polycythaemia rubra vera to myelofibrosis and appear to be associated with rapid disease progression. ${ }^{125}$ In one report multiple painful osteolytic lesions developed rapidly in a patient diagnosed as having myelofibrosis 18 years earlier. $^{6}$

Biopsy specimens taken from such lesions may show a range of appearances with trilineage hyperplasia, fibrosis or immature cells of the myeloid, and/or megakaryocytic series. ${ }^{5}$

There has been a report of osteosclerotic bone lesions being mistaken for metastatic carcinoma in a patient with breast carcinoma, who was subsequently found to have myelofibrosis. ${ }^{7}$ Another patient with myelofibrosis and breast carcinoma developed multiple osteolytic bone lesions shortly before her death. These lesions were subsequently shown to be due to marrow hyperplasia. ${ }^{8}$ However, the finding of a single, large, painless osteolytic lesion in a patient known to have both longstanding stable myelofibrosis and a carcinoma appears unusual and initially caused concern regarding the possibility of metastatic bronchial carcinoma. This was discounted after histological examination of the lesion.

The length of time between the diagnosis of myelofibrosis and the finding of the osteolytic lesion (33 years) and the stability of the myelofibrosis are also unusual. Other reported cases have been in patients with progressive disease of shorter duration.

Our patient later died of overwhelming postsplenectomy infection despite complying with postsplenectomy antibiotic prophylaxis. Overwhelming infection in splenectomised patient is a well recognised complication and its occurrence 13 years postoperatively in a patient on prophylactic antibiotics is a salutary reminder of the necessity to treat all postsplenectomy patients who develop infection vigorously with broad spectrum antibiotics including cover for capsulate organisms.

1 Kosmidis PA, Palacas CG, Axelrod AR. Diffuse purely osteolytic lesions in myelofibrosis. 7 Cancer 1980;46:2263-5. Leimert JT, Armitage JO, Dick FR. Myeloid metaplasia and Leimert JT, Armitage JO, Dick FR. Myeloid metaplasia
osteolytic lesions. Am $\mathcal{f}$ Clin Pathol 1978;70:706-8.

3 Weinstein IM. Idiopathic myelofibrosis: historical review, diagnosis and management. Blood Rev 1991;5:98-104.

4 Cho K, Bennet B. Correlation conferences in radiology and pathology. NY State f Med 1982;82:46-9.

5 Cassi E, De Paoli A, Tosi A, Turri C. Pure osteolytic lesions in myelofibrosis: report of two cases. $\mathcal{F}$ Haematol 1985;70: 178-83.

6 Case records of the Massachusetts General Hospital-Case 65. N Engl f Med 1963;269:854-63.

7 Peison B, Benisch B. Malignant myelosclerosis simulating metastatic bone disease. Radiology 1977;125:62.

8 Case records of the Massachusetts General Hospital-Case 39. N Engl f Med 1974;280:721-30. 